FACTA UNIVERSITATIS (NIŠ)

Ser. Math. Inform. Vol. 35, No 2 (2020), 351-356

https://doi.org/10.22190/FUMI2002351B

\title{
INEQUALITIES FOR GRADIENT EINSTEIN AND RICCI SOLITONS
}

\author{
Adara-Monica Blaga and Mircea Crasmareanu
}

(c) 2020 by University of Niš, Serbia | Creative Commons Licence: CC BY-NC-ND

Abstract. This short note concerns with two inequalities in the geometry of gradient Einstein solitons $(g, f, \lambda)$ on a smooth manifold $M$. These inequalities provide some relationships between the curvature of the Riemannian metric $g$ and the behavior of the scalar field $f$ through two quadratic equations satisfied by the scalar $\lambda$. The similarity with gradient Ricci solitons and a slight generalization involving a $g$-symmetric endomorphism $A$ are provided.

Keywords: gradient Einstein solitons; smooth manifold; Riemannian metric; $g$-symmetric endomorphism.

\section{Introduction}

Let $\left(M^{n}, g\right)$ be an $n$-dimensional Riemannian manifold endowed with a smooth function $f \in C^{\infty}(M)$; an excellent textbook in Riemannian geometry is [6]. The scalar field $f$ yields the Hessian endomorphism: $h_{f}: \mathfrak{X}(M) \rightarrow \mathfrak{X}(M), h_{f}(X)=$ $\nabla_{X} \nabla f$, where $\nabla$ is the Levi-Civita connection of $g$. Then we know the symmetry of the Hessian tensor field of $f: H_{f}(X, Y):=g\left(h_{f}(X), Y\right)$, namely $H_{f}(X, Y)=$ $H_{f}(Y, X)$. What follows is the existence of a $g$-orthonormal frame field $E=$ $\left\{E_{i}\right\}_{i=1, \ldots, n} \subset \mathfrak{X}(M)$ and the existence of the eigenvalues $\lambda=\left\{\lambda_{i}\right\}_{i=1, \ldots, n} \subset$ $C^{\infty}(M)$ :

$$
h_{f}\left(E_{i}\right)=\lambda_{i} E_{i}
$$

Hence we express all the geometric objects related to $f$ in terms of the pair $(E, \lambda)$ which we call the spectral data of $f$ :

$$
\nabla f=\sum_{i=1}^{n} E_{i}(f) E_{i}, \quad\|\nabla f\|_{g}^{2}=\sum_{i=1}^{n}\left[E_{i}(f)\right]^{2}, \quad h_{f}(X)=\sum_{i=1}^{n}\left(\lambda_{i} X^{i}\right) E_{i}
$$

Received June 06, 2018; accepted July 13, 2019

2010 Mathematics Subject Classification. Primary 53C25, 53C44, 53C21 
for $X=\sum_{i=1}^{n} X^{i} E_{i}$. Also the Hessian and the Laplacian of $f$ are:

$$
H_{f}(X, Y)=\sum_{i=1}^{n} \lambda_{i}\left(X^{i} Y^{i}\right), \quad \Delta f:=\operatorname{Tr}_{g} H_{f}=\sum_{i=1}^{n} \lambda_{i} .
$$

Let us remark that if $\nabla f$ does not have zeros and $E_{1}$ is exactly its unit vector field i.e. $E_{1}=\frac{\nabla f}{\|\nabla f\|_{g}}$, then $\nabla f$ is a geodesic vector field: $\nabla_{\nabla f} \nabla f=\lambda_{1} \nabla f$ which means that the flow of $\nabla f$ consists in geodesics of $g$.

\section{Results}

Assume now that the triple $(g, f, \lambda \in \mathbb{R})$ is a gradient Einstein soliton on $M,[2$, p. 67]:

$$
H_{f}+R i c+\left(\lambda-\frac{R}{2}\right) g=0
$$

where Ric is the Ricci tensor field of $g$ and $R$ is the scalar curvature. Einstein solitons generate self-similar solutions of the Einstein flow (1.1) of [2] and are more rigid than the well-known Ricci solitons. By considering the Ricci endomorphism $Q \in \mathcal{T}_{1}^{1}(M)$ provided by:

$$
\operatorname{Ric}(X, Y)=g(Q X, Y),
$$

we can express (2.1) as:

$$
h_{f}+Q+\left(\lambda-\frac{R}{2}\right) I=0
$$

with $I$ the Kronecker endomorphism. From (2.3) we get that $Q$ is also of diagonal form with respect to the frame $E$ :

$$
Q(X)=-\sum_{i=1}^{n}\left(\lambda_{i}+\lambda-\frac{R}{2}\right) X^{i} E_{i}, \quad\|Q\|_{g}^{2}=\sum_{i=1}^{n}\left(\lambda_{i}+\lambda-\frac{R}{2}\right)^{2} .
$$

By developing the second formula above we derive:

$$
\begin{gathered}
\|R i c\|_{g}^{2}=\sum_{i=1}^{n} \lambda_{i}^{2}+(2 \lambda-R) \sum_{i=1}^{n} \lambda_{i}+n\left(\lambda^{2}-\lambda R+\frac{R^{2}}{4}\right)= \\
=\left\|H_{f}\right\|_{g}^{2}+(2 \lambda-R) \Delta f+n\left(\lambda^{2}-\lambda R+\frac{R^{2}}{4}\right) .
\end{gathered}
$$

Hence the scalar $\lambda$ is a solution of the quadratic equation:

$$
n \lambda^{2}+2\left(\Delta f-\frac{n R}{2}\right) \lambda+\left(\left\|H_{f}\right\|_{g}^{2}-\|R i c\|_{g}^{2}+\frac{n R^{2}}{4}-R \Delta f\right)=0
$$

which means the non-negativity:

$$
0 \leq \Delta^{\prime}:=\left(\Delta f-\frac{n R}{2}\right)^{2}-n\left(\left\|H_{f}\right\|_{g}^{2}-\|R i c\|_{g}^{2}+\frac{n R^{2}}{4}-R \Delta f\right) .
$$


It follows a lower boundary of the geometry of $g$ in terms of $f$ :

$$
\|R i c\|_{g}^{2} \geq\left\|H_{f}\right\|_{g}^{2}-\frac{1}{n}(\Delta f)^{2}
$$

An "exotic" consequence is provided by the case of strict inequality in (2.7), more precisely, it follows that the data $(g, f, \lambda)$ is doubled by $\left(g, f, \frac{2 \Delta f}{n}-R-\lambda=-\frac{2}{n} R-\lambda\right)$.

Example 1 i) (Gaussian soliton) We have $\left(M=\mathbb{R}^{n}, g_{\text {can }}\right)$ and $f(x)=-\frac{\lambda}{2}\|x\|^{2}$. It results $h_{f}=-\lambda I_{n}$ and $\Delta f=-n \lambda$. Since $\left\|H_{f}\right\|^{2}=n \lambda^{2}$, the left hand side of (2.6) is:

$n \lambda^{2}+2\left(\Delta f-\frac{n R}{2}\right) \lambda+\left(\left\|H_{f}\right\|_{g}^{2}-\|R i c\|_{g}^{2}+\frac{n R^{2}}{4}-R \Delta f\right)=n \lambda^{2}+2(-n \lambda) \lambda+n \lambda^{2}$

which is exactly zero. Also: $\Delta^{\prime}=(n \lambda)^{2}-n\left(n \lambda^{2}-0\right)=0$ which means the uniqueness of $\lambda$ and the equality case in (2.8): $0=n \lambda^{2}-\frac{(n \lambda)^{2}}{n}$.

ii) A generalization of the previous example is provided on a Ricci-flat manifold by a smooth function $f$ satisfying a generalization of Hessian structures:

$$
H_{f}=-\lambda g \text {. }
$$

Then $\Delta f=-n \lambda$ and $\left\|H_{f}\right\|^{2}=n \lambda^{2}$ exactly as for the Gaussian soliton. Using Lemma 4.1. of [3, p. 1540] it results form (2.9) that $\nabla f$ is a particular concircular vector field: $h_{f}=-\lambda I$; hence $\lambda_{1}=\ldots=\lambda_{n}=-\lambda$ is the spectral part of the spectral data of $f$. If $\nabla f$ is without zeros it follows from Theorem 3.1. of [3, p. 1539 ] that $(M, g)$ is locally a warped product manifold with a 1-dimensional basis: $(M, g)=\left(I \subseteq \mathbb{R}, g_{c a n}\right) \times_{\varphi}\left(F^{n-1}, g_{F}\right)$. In fact, $\nabla f=\varphi(s) \frac{\partial}{\partial s}$ with $\varphi^{\prime}(s)=-\lambda$ which means an affine warping function, $\varphi(s)=-\lambda s+C$.

A new quadratic equation, similar to (2.6), follows from:

$$
\Delta f+\left(1-\frac{n}{2}\right) R+n \lambda=0
$$

obtained by tracing (2.1). Hence the companion equation of (2.6) is:

$$
n \lambda^{2}+2\left(1-\frac{n}{2}\right) R \lambda+\left(\|R i c\|_{g}^{2}-\left\|H_{f}\right\|_{g}^{2}+\frac{n-4}{4} R^{2}\right)=0 .
$$

The new inequality is then:

$$
0 \leq \Delta^{\prime}:=\left(1-\frac{n}{2}\right)^{2} R^{2}-n\left(\|R i c\|_{g}^{2}-\left\|H_{f}\right\|_{g}^{2}+\frac{n-4}{4} R^{2}\right)
$$

and it results a lower boundary of the behavior of $f$ in terms of the geometry of $g$ :

$$
\left\|H_{f}\right\|_{g}^{2} \geq\|R i c\|_{g}^{2}-\frac{R^{2}}{n}=\frac{1}{n} \sum_{i \neq j}\left(\lambda_{i}-\lambda_{j}\right)^{2} .
$$


We remark that (2.8) and (2.13) can be unified in the double inequality:

$$
\left\|H_{f}\right\|_{g}^{2}-\frac{1}{n}(\Delta f)^{2} \leq\|R i c\|_{g}^{2} \leq\left\|H_{f}\right\|_{g}^{2}+\frac{R^{2}}{n}
$$

and the simultaneous equalities for $n \geq 3$ hold if and only if $R=\Delta f=0=\lambda$ and $H_{f}=-R i c$; hence $f$ is a harmonic map on a steady gradient Einstein soliton. The vanishing of the right-hand side of (2.13) means that $g$ is an Einstein metric; other interesting aspects concerning the functional $F_{g}:=\frac{R^{2}}{\|R i c\|_{g}^{2}}$ on the space of non-flat metrics appear in [5]. This raises the first future problem to study the similar functional $F_{f}^{g}:=\frac{(\Delta f)^{2}}{\left\|H_{f}\right\|_{g}^{2}}$ on the space of smooth functions which are not linear on $M$ after the name from [6, p. 283]. Remark that for the Hessian structures (2.9) we have a constant and maximal $F_{f}^{g}=n$.

Example 1 revisited i) (Gaussian soliton) The inequality (2.13) becomes $n \lambda^{2} \geq 0$.

ii) Again, (2.13) means $n \lambda^{2} \geq 0$.

iii) (relationship with gradient Ricci solitons) If $R=0$, then the gradient Einstein soliton becomes a gradient Ricci soliton and we remark that (2.14) is exactly the double inequality $(20)$ of $[4$, p. 3339]. The explication of this fact is provided by the following remark.

Remark An unified proof of the double inequality (2.14) is provided by the following relation satisfied by an Einstein soliton, which is a direct consequence of the equations (2.5) and (2.10):

$$
n\left(\left\|H_{f}\right\|_{g}^{2}-\|R i c\|_{g}^{2}\right)=(\Delta f)^{2}-R^{2}
$$

and it is important to point out that this equation does not involves the scalar $\lambda$. In other words, $(2.15)$ is a universal formula of the gradient Einstein solitons. With $\lambda \rightarrow \lambda+\frac{R}{2}$ we get that (2.15) holds also for gradient Ricci solitons and hence we obtain the similarity between gradient Ricci and Einstein solitons with respect to (2.14).

Returning to (2.3) we remark that the Ricci endomorphism $Q$ commutes with $h_{f}$ for an Einstein or Ricci gradient soliton. It results the commuting property also for the Einstein endomorphism:

$$
\text { Einst }_{g}:=Q-\frac{R}{n} I
$$

which is the trace-free part of $Q$. We will assume now that the data $(g, f, \lambda, \mu \in \mathbb{R})$ satisfies:

$$
h_{f}+Q+\lambda I+\mu \text { Einst }_{g}=0 .
$$

The corresponding relation in terms of Ricci endomorphism is:

$$
h_{f}+(1+\mu) Q+\left(\lambda-\frac{\mu R}{n}\right) I=0
$$


or, for $\mu \neq-1$ :

$$
h_{\frac{f}{1+\mu}}+Q+\left(\frac{\lambda}{1+\mu}-\frac{\mu R}{n(1+\mu)}\right) I=0 .
$$

This last equation is an example of $\rho$-Einstein soliton as is introduced in Definition 1.1 of $\left[2\right.$, p. 67] with $\rho=\frac{\mu}{n(1+\mu)}$ and $(f, \lambda)$ of [2] replaced by $\frac{1}{1+\mu}(f, \lambda)$.

Hence we naturally arrive to the following slight generalization of all the above considerations. Fix a $g$-symmetric endomorphism $A \in \mathcal{T}_{1}^{1}(M)$ which is also diagonal with respect to the frame $E$ :

$$
A\left(E_{i}\right)=\rho_{i} E_{i}, \quad \rho_{i} \in C^{\infty}(M) .
$$

Hence $A$ and $h_{f}$ commutes: $A \circ h_{f}=h_{f} \circ A$. We introduce:

Definition The data $(g, f, \lambda, \mu \in \mathbb{R})$ is an A-Ricci gradient soliton if:

$$
h_{f}+Q+\lambda I+\mu A=0 .
$$

We get that $A$ commutes also with $Q$ and the corresponding generalization of $(2.15)$ is:

$(2.22) n\left[\left\|H_{f}\right\|_{g}^{2}-\|R i c\|_{g}^{2}+\mu^{2}\|A\|_{g}^{2}+2 \mu \operatorname{Tr}_{g}\left(h_{f} \circ A\right)\right]=\left(\Delta f+\mu T r_{g} A\right)^{2}-R^{2}$

yielding the double inequality:

$$
\begin{gathered}
\left\|H_{f}\right\|_{g}^{2}-\frac{1}{n}\left(\Delta f+\mu \operatorname{Tr}_{g} A\right)^{2}+\mu^{2}\|A\|_{g}^{2}+2 \mu \operatorname{Tr}_{g}\left(h_{f} \circ A\right) \leq\|R i c\|_{g}^{2} \leq \\
\leq\left\|H_{f}\right\|_{g}^{2}+\frac{R^{2}}{n}+\mu^{2}\|A\|_{g}^{2}+2 \mu \operatorname{Tr}_{g}\left(h_{f} \circ A\right) .
\end{gathered}
$$

There is another problem: to find remarkable endomorphisms commuting with a given $h_{f}$. We will finish this note with an example.

Example 2 Suppose that $(M, g)$ is a hypersurface in $\left(N^{n+1}, g\right)$ and let $A=S$ be the shape endomorphism of $M$ commuting with $h_{f}$ for the fixed scalar field $f \in C^{\infty}(M)$. If $(g, f, \lambda, \mu \in \mathbb{R})$ is a shape-Ricci gradient soliton on $M$ i.e. (2.21) holds for $S$, then denoting by $H$ the mean curvature of $M$, we get:

$$
\begin{gathered}
\left\|H_{f}\right\|_{g}^{2}-\frac{1}{n}(\Delta f+\mu H)^{2}+\mu^{2}\|S\|_{g}^{2}+2 \mu T r_{g}\left(h_{f} \circ S\right) \leq\|R i c\|_{g}^{2} \leq \\
\leq\left\|H_{f}\right\|_{g}^{2}+\frac{R^{2}}{n}+\mu^{2}\|S\|_{g}^{2}+2 \mu T r_{g}\left(h_{f} \circ S\right) .
\end{gathered}
$$

We point out that immersions of (almost) Ricci solitons into another Riemannian manifold are studied in [1]. 


\title{
REFER E N C ES
}

1. A. Barros, J. N. Gomes AND E. RiBeIro: Immersion of almost Ricci solitons into a Riemannian manifold, Mat. Contemp., 40 (2011), 91-102.

2. G. Catino And L. Mazzieri: Gradient Einstein solitons, Nonlinear Anal. Ser. A Theory Methods, 132 (2016), 66-94.

3. B.-Y. CHEN: Some results on concircular vector fields and their applications to Ricci solitons, Bull. Korean Math. Soc., 52 (2015), no. 5, 1535-1547.

4. M. Crasmareanu: A new approach to gradient Ricci solitons and generalizations, Filomat, 32 (2018), no. 9, 3337-3346.

5. J. LAURet AND Cynthia E. Will: The Ricci pinching functional on solvmanifolds, Quart. J. Math., 70 (2019), no. 4, 1281-1304.

6. P. Petersen: Riemannian geometry, Third edition. Graduate Texts in Mathematics 171, Springer, Cham, 2016.

\author{
Adara-Monica Blaga \\ West University of Timişoara \\ Department of Mathematics \\ Bd. V. Parvan, no. 4 \\ 300223, Timişoara, România \\ adarablaga@yahoo.com \\ Mircea Crasmareanu \\ University "Al. I. Cuza" \\ Department of Mathematics \\ Bd. Carol I, no. 11 \\ 700506, Iaşi, România \\ mcrasm@uaic.ro
}

